\title{
A Rapid Screening Method for Sibutramine Hydrochloride in Natural Herbal Medicines and Dietary Supplements
}

\author{
Qi Liang $\mathbb{D}^{1},{ }^{1}$ Yue Zhuang, ${ }^{1}$ Jun Ma, ${ }^{1}$ Jinyan Wang, ${ }^{1}$ Rui Feng, ${ }^{2}$ Ruisi He, ${ }^{3}$ Zhuoya Luo $\mathbb{D}^{1}$ \\ Honghao Wang, ${ }^{4}$ and Ruoting Zhan ${ }^{5}$ \\ ${ }^{1}$ Guangdong Institute for Drug Control, Guangzhou, China \\ ${ }^{2}$ Research Center of Chinese Herbal Resource Science and Engineering, Guangzhou University of Chinese Medicine, \\ Guangzhou, China \\ ${ }^{3}$ School of Pharmaceutical Sciences, Sun Yat-Sen University, Guangzhou, China \\ ${ }^{4}$ Market Regulation Administration of Guangdong, Guangzhou, China \\ ${ }^{5}$ School of Chinese Materia Medica, Ministry of Education, Key Laboratory of Chinese Medicinal Resource from Lingnan, \\ Guangzhou University of Chinese Medicine, Guangzhou, China
}

Correspondence should be addressed to Zhuoya Luo; luozhuoya@gdidc.org.cn

Received 3 June 2020; Revised 25 April 2021; Accepted 31 July 2021; Published 26 August 2021

Academic Editor: Maria Jose Ruiz Angel

Copyright $\odot 2021$ Qi Liang et al. This is an open access article distributed under the Creative Commons Attribution License, which permits unrestricted use, distribution, and reproduction in any medium, provided the original work is properly cited.

Herbal weight loss drugs are becoming more widely used in the fight against obesity, but ineffective regulation of these products have resulted in harmful additives. These products may contain adulterants such as sibutramine hydrochloride that may result in serious adverse health events including death. This work established a color precipitation reaction-based rapid screening method for illegal adulteration of sibutramine hydrochloride in natural herbal medicines (NHM) and dietary supplements (DS). While a variety of chromatography- and electrophoresis-based systems have been reported to measure this analyte, they generally suffer from high costs, complicated sample preparation, and a costly analytical infrastructure. In contrast, we present a simple, handheld kit to assay for sibutramine. The performance metrics of this tool include an average detection time of approximately 3 minutes, which is markedly shorter than conventional methods (HPLC or HPLC-MS, etc.), a detection limit of $0.1 \mathrm{mg}$ per aliquot, and an accuracy of $99.02 \%(n=820)$. More strikingly, the sensitivity is $100 \%(n=278)$, and the specificity is $98.52 \%(n=542)$. The rapid test kit developed from this screening method was evaluated by FDA. In summary, this screening method is a rapid, simple, and low-cost tool for the detection of sibutramine in NHM and DS with superior selectivity and sensitivity. For these reasons, this method is especially suitable for underdeveloped settings because it can be employed onsite without any instrumentation. In addition, this approach could rapidly exclude most of the negative samples to boost efficiency in large-scale samples assay. If necessary, positive samples can undergo further alternate testing methods to confirm the positive results of sibutramine hydrochloride content.

\section{Introduction}

Sibutramine hydrochloride, $\quad \mathrm{N}-\{1$-[1-(4-chloroph-enyl) cyclobut-yl]-3-methylbutyl\}-N,N-dim-ethylamine hydrochloride monohydrate $\left(\mathrm{C}_{17} \mathrm{H}_{26} \mathrm{ClN} \cdot \mathrm{HCl} \cdot \mathrm{H}_{2} \mathrm{O}\right)$ is a reuptake inhibitor of noradrenaline and 5-hydroxytryptamine and has been used as an antiobesity drug since 1997 [1]. It has a molecular weight of $334.33 \mathrm{~g} / \mathrm{mol}$.

Sibutramine hydrochloride may cause severe adverse effects including insomnia, psychosis, affective psychosis, panic attacks, delirious state, amnesia, bipolar disorder, hypomanic or manic episodes, and increases the risk of stroke and heart attack [2-15]. Consequently, FDA has withdrawn sibutramine hydrochloride from the US market in October 2010 [16].

Recently, illegal adulteration of natural herbal medicine (NHM) and dietary supplements (DS) with sibutramine hydrochloride has been reported and is dangerous for consumers.

To date, numerous detection methods for sibutramine hydrochloride adulteration in NHM and DS for weight loss have been developed including thin-layer chromatography 
(TLC) [17, 18], gas chromatography mass spectrometry (GC-MS) [19, 20], liquid chromatography (LC) [21, 22], liquid chromatography-mass spectrometry (LC-MS) [21, 23-27], ion exchange chromatography (IEC) [28], capillary electrophoresis (CE) [29], capillary zone electrophoresis (CZE) [30], nuclear magnetic resonance (NMR) [31], ion mobility spectrometry (IMS) [32], infrared spectroscopy (IR) [33], micellar electrokinetic capillary chromatography (MECC) [34], X-ray powder diffraction (XRPD), and colorimetry $[35,36]$. However, there are several disadvantages of these methods including long assay time, expensive instrumentation, and the need for skilled operators. As a result, these methods are an obstacle to consistent and cost-effective testing of NHM and DS.

Color tests have been widely used in drug analysis historically. They are simple, inexpensive, and easy to operate. The results of these methods are easily distinguished and commonly used to assist the identification. Without the precise but unwieldy instruments, the color tests can provide a preliminary estimate of the samples at the various scenes. These results are not as accurate as the instrument analysis, but effective enough for on-site inspection [37-40].

Therefore, this work establishes a rapid, simple, colorimetric screening method for sibutramine hydrochloride in NHM and DS. This method can be developed as a rapid screening kit for onsite analysis of sibutramine hydrochloride in NHM and DS. The method could also be an initial rapid screen prior to instrumental analysis.

\section{Materials and Methods}

2.1. Instrumentation. Millipore Milli-Q water purification system (Millipore, USA) and LC-20A HPLC system with UV detector (Shimadzu, Japan) were used.

2.2. Reference Standard and Reagents. Sibutramine hydrochloride monohydrate (National Institutes for Food and Drug Control, China; Lot. no. 100624-200401, desiccated for two hours at $105^{\circ} \mathrm{C}$ before use). Ethyl acetate (AR, Guangzhou Chemical Reagent Co.), phosphoric acid (analytical grade, Guangzhou Chemical Reagent Co.), ammonium reineckate (analytical grade, Shanghai Hengxin Chemical reagent Co., Ltd.), methanol (HPLC grade, Guangzhou Chemical Reagent Co.), triethylamine (analytical grade, Guangzhou Chemical Reagent Co.), acetonitrile (HPLC grade, Merck \& Co., Inc., USA), and sodium heptanesulfonate (analytical grade, Shandong Yuwang Industrial Co., Ltd.) were used in this study.

2.3. Samples. Zhuoyue Fengzi Slimming Capsule and Fufang Shoubao Capsule were used, courtesy of Guangdong Institute for Drug Control.

2.4. Preparation of Ammonium Reineckate Detection Reagent. $0.1 \mathrm{~g}$ of ammonium reineckate is dissolved in $4 \mathrm{ml}$ of purified water and shaked for $1 \mathrm{~min}$. This solution should be freshly prepared and used within 48 hours.

\subsection{Rapid Screening Method Procedure}

(i) Powdered sample ( 0.1 to $0.5 \mathrm{~g}$ ) was placed in a $7 \mathrm{ml}$ sample vial (A). We then added $3.5 \mathrm{ml}$ of ethyl acetate to vial $\mathrm{A}$, inverted it 5 times, and allowed it to stand for $1 \mathrm{~min}$.

(ii) We used a burette to extract $2 \mathrm{ml}$ of the upper-layer liquid (organic phase) of vial A and transferred it to another sample vial (B). We then added $3 \mathrm{ml}$ of $3 \%$ $\mathrm{H}_{3} \mathrm{PO}_{4}$ to vial $\mathrm{B}$. This was inverted 5 times to make a dispersed phase and allowed to stand for $1 \mathrm{~min}$.

(iii) Next, we used a burette to extract $1 \mathrm{ml}$ of the lowerlayer liquid (aqueous phase) from vial $B$ and transferred it to a test tube. We then added 1 drop of ammonium reineckate detection reagent. If an obvious light-pink insoluble substance is formed immediately, then the sample is positive for sibutramine. Otherwise, it is negative.

2.6. LC Determination of Sibutramine. An LC-20A HPLC system (Shimadzu, Japan) equipped with an automatic sampler and a UV detector was used for the chromatographic analysis. A KQ-300DE ultrasonic generator was purchased from Kunshan Ultrasonic Instruments Co., Ltd., China.

The chromatographic analysis was carried out in isocratic mode on an ODS-2 Hypersil $\mathrm{C}_{18}$ analytical column $(250 \mathrm{~mm} \times 4.6 \mathrm{~mm} ; 5 \mu \mathrm{m}$ particle size; Thermo Fisher Scientific, USA). The mobile phase in the HPLC method was methanol-acetonitrile-sodium heptanesulfonate solution (10: $3: 7, \mathrm{v} / \mathrm{v} / \mathrm{v})$. Sodium heptanesulfonate solution was prepared as follows: add $1000 \mathrm{ml}$ of water in a beaker, dissolve $2.02 \mathrm{~g}$ of sodium heptanesulfonate, and then add $0.8 \mathrm{ml}$ of triethylamine and adjust the $\mathrm{pH}$ to $3.3 \pm 0.1$ with glacial acetic acid. The flow rate was $1.0 \mathrm{ml} / \mathrm{min}$, the detection wavelength was $223 \mathrm{~nm}$, and the injection volume was $20 \mu \mathrm{l}$.

$10 \mathrm{mg}$ of sibutramine hydrochloride was weighed and dissolved in $50 \mathrm{ml}$ of mobile phase. It was then gradually diluted to $0.2 \mathrm{mg} / \mathrm{ml}$ in mobile phase with volumetric pipettes and flasks. Quality control solutions were prepared from independent stock standards at 0.5, 1.0, 2.0, 25, 50, 80, and $100 \mu \mathrm{g} / \mathrm{ml}$ in mobile phase. Each quality control solution was filtered through a Sartorius model $0.45 \mu \mathrm{m}$ polytetrafluoroethylene (PTFE) filter before application in HPLC system. The calibration curve was linear from 0.536 to $107.2 \mu \mathrm{g} / \mathrm{ml}\left(Y=40939 X+2517.6, R^{2}=1\right)$.

\section{Results and Discussion}

3.1. Principle of the Rapid Screening Method. The outermost electron orbital of the nitrogen atom in sibutramine is a $\mathrm{SP}^{3}$ hybrid. Thus, there are two lone-paired electrons in the $s$ orbital, which approach the empty orbital of the proton in inorganic acid solutions and form an ammonium cation with proton. As the nitrogen atom combines with high $\mathrm{pKa}$ alkyls ( $\left.\mathrm{pKa}_{\mathrm{CH} 4}=49\right)$, it donates electron part of the positive charge of the ammonium cation that can be dispersed to attain stability. Thus, sibutramine tends to be protonated and well solubilized in acid solution (Figure 1(a)) [41]. 


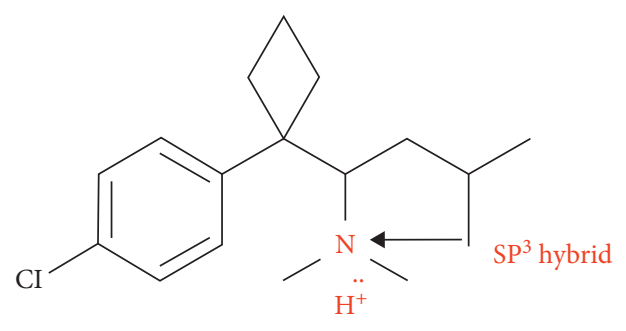

(a)

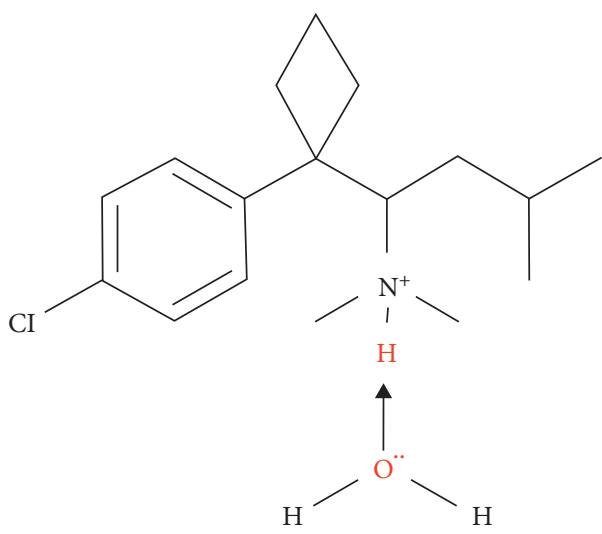

(b)

FIgUre 1: Schematic of dissolution in acid aqueous solution of sibutramine. (a) Protonation of sibutramine. (b) Solvation of sibutramine.

Due to the electronegativity of the nitrogen atom in protonated sibutramine, it combines with the nitrogen atom to form a hydrogen bond with water and become solvated (Figure 1(b)). Therefore, sibutramine hydrochloride can easily be dissolved in inorganic acid aqueous solution. On the other hand, as it has a benzene ring and an aliphatic amine structure, sibutramine can also easily dissolve in organic solvents such as methanol, ethanol, $n$-butanol, ethyl acetate, benzene, and dichloromethane.

NHM and DS usually consist of multiple compounds, most of which are colored substances such as phytochromes. Thus, it is necessary to remove these substances before initiating the color reaction for rapid screening because the colored substances may interfere with the colored reaction.

We developed a two-step extraction protocol for sibutramine hydrochloride because of its good solubility in both organic acid solvents and inorganic solution. First, an organic solvent is applied to extract sibutramine hydrochloride from samples, which separates the interfering substances that are insoluble in organic solvents. Second, a liquid-liquid extraction (LLE) is performed with an inorganic acid to further separate sibutramine from the organic solvent that contains the interfering substances.

There are three criteria in selecting the sibutramine hydrochloride extraction buffer: (1) it must dissolve sibutramine hydrochloride; (2) it may not dissolve the inorganic acid solution; and (3) it should be environmentally friendly with no human toxicity. Fortunately, there are numerous organic solvents that meet these criteria including ethyl acetate with good extraction effect and low toxicity.

Both ethyl acetate and sibutramine are electron-donating compounds. They cannot form a hydrogen bond; however, they can attract each other with Van der Waals forces to form weak interactions. Thus, ethyl acetate can easily dissolve and extract sibutramine because they have similar polarities. In addition, ethyl acetate can exclude insoluble substances that may potentially interfere with sibutramine assay.

More importantly, LLE can protonate, solvate, and extract sibutramine and also exclude most competing substances that are insoluble in acid aqueous medium and may interfere with the test results. While many acids could be used, we selected $\mathrm{H}_{3} \mathrm{PO}_{4}$ because of its noncorrodibility, nonvolatility, and convenience.

The sibutramine is protonated on contact with inorganic acid. The polarity of the protonated sibutramine increases, so the difference in polarities between the protonated sibutramine and the ethyl acetate increases, and thus their affinity decreases. Protonated sibutramine then forms hydrogen bonds with water and is solvated and then moves into the inorganic phase from the organic phase.

Sibutramine accepts a proton to form a quaternary ammonium salt $\left(\mathrm{R}^{+}\right)$, and the detection agent should precipitate with the quaternary ammonium salt. For this, we chose ammonium reineckate, which is soluble in water and produces a cation of $\mathrm{NH}_{4}^{+}$and an anion of $\left[\mathrm{Cr}\left(\mathrm{NH}_{3}\right)_{2}(\mathrm{SCN})_{4}\right]^{-}$.

The cation of $\mathrm{R}^{+}$reacts with the anion of $\left[\mathrm{Cr}\left(\mathrm{NH}_{3}\right)_{2}(\mathrm{SCN})_{4}\right]^{-}$to form an organic salt that is insoluble in acidic aqueous medium. The organic salt is pink, and the precipitate is easily visualized. The ion reaction formula is described in the following equation:

$$
R^{+}+\left[\mathrm{Cr}\left(\mathrm{NH}_{3}\right)_{2}(\mathrm{SCN})_{4}\right]^{-} \longrightarrow R\left[\mathrm{Cr}\left(\mathrm{NH}_{3}\right)_{2}(\mathrm{SCN})_{4}\right] \downarrow
$$

3.2. Optimization of the Method. The main influencing factors of the method were the property and amount of the extraction liquids. To select ethyl acetate and phosphoric acid solution as the extraction liquid has been briefly explained in the above. Based on these results, further optimization of the method was undertaken as follows.

3.2.1. Concentration of Phosphoric Acid Solution. The high efficiency of the extraction step is important because the violent shaking, repeated steps, and long layering time are not always allowed in the rapid screening methods to enhance the performance. This study used dilute phosphoric acid solution and measured the partition coefficient of sibutramine hydrochloride in ethyl acetate and diluted phosphoric acid to optimize the condition. 
The partition coefficient was estimated by the following equation:

$$
K=\frac{C_{A}}{C_{B}}
$$

Here, both $C_{A}$ and $C_{B}$ are the concentrations of the test substance in two immiscible solvents while the distribution system becomes balanced.

$71.025 \mathrm{mg}$ of sibutramine hydrochloride standard was weighed and transferred into a $200 \mathrm{ml}$ volumetric flask. Then, ethyl acetate was added to dissolve the sample and diluted to the volume. Next, $10.0 \mathrm{ml}$ of the solvent was transferred into a $60 \mathrm{ml}$ separation funnel. This was repeated for 11 more samples. Each held $10.0 \mathrm{ml}$ of diluted phosphoric acid at $0.5 \%, 1.0 \%, 3.0 \%$, and $5.0 \%$, respectively. The flask was sealed, shaken, and drained. This was repeated and allowed to stand for layering. We next determined the content of the sibutramine hydrochloride in this two-phase solution with HPLC.

We removed $0.5 \mathrm{ml}$ from the supernatant and the lower layer and placed them in two $10 \mathrm{ml}$ volumetric flasks, respectively. After adding mobile phase to each, we mixed the samples, filtered, and analysed them both with HPLC (Table 1). As shown in Table 1, the concentration variation of the diluted phosphate solution had little effect on the partition coefficients. The coefficients increased slightly with the concentration rising and reached the maximum value when the concentration of the diluted phosphate solution was around 3.0\%. Therefore, a 3.0\% diluted phosphate solution was chosen as the extraction solution.

3.2.2. Volume of the Extraction Liquid. An orthogonal experiment was performed to optimize the volume of extraction liquids in each step. The concentration of the sample used in this experiment was $0.86 \mathrm{mg} / \mathrm{g}$, which was determined by HPLC at first. The experiment operated by the following steps:

(i) Powdered sample $(0.15 \mathrm{~g})$ was placed in a $10 \mathrm{ml}$ sample vial (A). Then, $V_{1} \mathrm{ml}$ ethyl acetate was added to vial $\mathrm{A}$, inverted it 5 times, and allowed it to stand for $1 \mathrm{~min}$. Determine the concentration of sibutramine hydrochloride in ethyl acetate $\left(C_{1} \mu \mathrm{g} / \mathrm{ml}\right)$ by HPLC.

(ii) $V_{2} \mathrm{ml}$ of the upper-layer liquid of vial $\mathrm{A}$ was transferred into another sample vial (B). $V_{3} \mathrm{ml} \mathrm{3 \%}$ $\mathrm{H}_{3} \mathrm{PO}_{4}$ was added to vial $\mathrm{B}$, inverted 5 times to make a dispersed phase and allowed to stand for $1 \mathrm{~min}$. By the way, the amount of $V_{3}$ was determined according to a series of fixed ratio of the two phases $\left(V_{2}: V_{3}\right)$. Determine the concentration of sibutramine hydrochloride in phosphoric acid solution $\left(C_{2} \mu \mathrm{g} / \mathrm{ml}\right)$ by HPLC.

As shown in Table 2, the extraction rates were about $40 \%$ when $2 \mathrm{ml}$ ethyl acetate was used. And the rates could be increased to about $80 \%$ with $3.5 \mathrm{ml}$ ethyl acetate. Furthermore, double the volume, the rates were increased about only 6 percent, but the concentration of the solution was nearly halved. In the LLE step, the rates were more than $90 \%$ in different conditions. It suggests that almost all the sibutramine was extracted into the phosphate acid solution. Considering the final step of the test, high concentration of the solution is favorable for the formation of the precipitate. Thus, the phosphate acid solution should be used as less as possible. However, there is a practical reason, the volume of the liquid used in rapid screening is better to be more than $2 \mathrm{ml}$ for easy operation and observation. Finally, $3.5 \mathrm{ml}$ ethyl acetate and $3 \mathrm{ml}$ phosphate acid solution was considered as the optimal condition.

3.2.3. Effects of Temperature. The effect of temperature on chromic reaction and LLE rate was studied by carrying out the rapid screening method under $10^{\circ} \mathrm{C}, 15^{\circ} \mathrm{C}, 20^{\circ} \mathrm{C}$, and $25^{\circ} \mathrm{C}$ (Table 3 ). At $10^{\circ} \mathrm{C}$, no visible pink precipitates are formed within $30 \mathrm{~s}$. At $15^{\circ} \mathrm{C}$ or higher temperature, pink precipitates are formed within $30 \mathrm{~s}$. The LLE rates at $10^{\circ} \mathrm{C}$, $15^{\circ} \mathrm{C}, 20^{\circ} \mathrm{C}$, and $25^{\circ} \mathrm{C}$ are $99.39 \%, 99.38 \%, 99.32 \%$, and $99.23 \%$ respectively.

3.3. Transfer Efficiency of the Method. By investigating the extraction rate and LLE rate, we demonstrate the transfer efficiency of sibutramine as well as overall feasibility. Extraction rate and LLE rate were determined by HPLC quantification of sibutramine in each extraction step.

The content powder removed from 20 Zhuoyue Fengzi Slimming Capsules was homogenously mixed and weighed. The mean mass was 0.285 g (R.S.D. $=2.76 \%, n=20$ ). We analytically weighed $0.1 \mathrm{~g}$ and added $50 \mathrm{ml}$ of the mobile phase for extraction accompanied with $10 \mathrm{~min}$ of treatment in an ultrasonic bath. The supernatant of the extraction solution was filtered through a $0.45 \mu \mathrm{m}$ PTFE filter before being applied to the HPLC system. The results include that average content of sample is $32.21 \mathrm{mg} / \mathrm{g}$ and RSD is $2.58 \%$ $(n=6)$; more details are shown in Table 4 .

$0.1 \mathrm{~g}$ of sample was transferred to a $10 \mathrm{ml}$ specimen bottle followed by $8.0 \mathrm{ml}$ of ethyl acetate and inversion 5 times. This was incubated at room temperature for $1 \mathrm{~min}$. We next transferred $3.0 \mathrm{ml}$ of the supernatant to another bottle for the LLE step (solution A). Here, we measured another $2.0 \mathrm{ml}$ of the supernatant and transferred it to a $25 \mathrm{ml}$ volumetric flask. Next, mobile phase was added to dilute to the mark followed by shaking. This was filtered and analyzed with HPLC. The results include that average extraction rate is $78.28 \%$ and RSD is $4.33 \%(n=6)$; more details are shown in Table 5 .

Procedure. Solution A was transferred to a $10 \mathrm{ml}$ bottle and mixed with $3.0 \mathrm{ml}$ of $3 \%$ phosphoric acid. The sample was inverted 5 times. We moved $1.0 \mathrm{ml}$ of the lower layer into a $10 \mathrm{ml}$ volumetric flask. Mobile phase was added to the mark and then mixed well. We filtered and measured the filtrate with HPLC. This was repeated for five more replicates and results in that average liquid-liquid extraction rate is $59.45 \%$ and RSD is $11.01 \%(n=6)$; more details are shown in Table 6.

The average transfer efficiency calculated according to the extraction rate and the LLE rate is $46.62 \%$ and RSD is 
TABLE 1: The results of partition coefficients.

\begin{tabular}{|c|c|c|c|c|c|c|}
\hline System* & Sample & Peak area & $C^{\mathrm{A}}$ or $C^{\mathrm{B}}(\mu \mathrm{g} / \mathrm{ml})$ & Partition coefficients $K$ & $K$ (avg.) & R.S.D. $\%(n=3)$ \\
\hline \multirow{6}{*}{1} & $\mathrm{~B}$ & 13958213 & 340.89 & \multirow{2}{*}{36.62} & \multirow{6}{*}{36.72} & \multirow{6}{*}{1.31} \\
\hline & A & 383660 & 9.31 & & & \\
\hline & B & 13936925 & 340.37 & \multirow{2}{*}{36.29} & & \\
\hline & $\mathrm{A}$ & 386525 & 9.38 & & & \\
\hline & B & 14138345 & 345.29 & \multirow{2}{*}{37.24} & & \\
\hline & A & 382022 & 9.27 & & & \\
\hline \multirow{6}{*}{2} & B & 13961488 & 340.97 & \multirow{2}{*}{42.00} & \multirow{6}{*}{40.37} & \multirow{6}{*}{3.51} \\
\hline & A & 334942 & 8.12 & & & \\
\hline & B & 14056876 & 343.30 & \multirow{2}{*}{39.64} & & \\
\hline & A & 357049 & 8.66 & & & \\
\hline & B & 14024944 & 342.52 & \multirow{2}{*}{39.47} & & \\
\hline & A & 357868 & 8.68 & & & \\
\hline \multirow{6}{*}{3} & B & 14275491 & 348.64 & \multirow{2}{*}{45.18} & \multirow{6}{*}{46.70} & \multirow{6}{*}{3.18} \\
\hline & $\mathrm{A}$ & 318567 & 7.72 & & & \\
\hline & B & 14247243 & 347.95 & 1814 & & \\
\hline & A & 298507 & 7.23 & 48.14 & & \\
\hline & B & 14171506 & 346.10 & \multirow{2}{*}{46.77} & & \\
\hline & $\mathrm{A}$ & 305466 & 7.40 & & & \\
\hline \multirow{6}{*}{4} & $\mathrm{~B}$ & 14118285 & 344.80 & \multirow{2}{*}{46.67} & \multirow{6}{*}{47.04} & \multirow{6}{*}{2.78} \\
\hline & A & 305057 & 7.39 & & & \\
\hline & B & 14198116 & 346.75 & \multirow{2}{*}{48.50} & & \\
\hline & A & 295231 & 7.15 & & & \\
\hline & B & 14093721 & 344.20 & \multirow{2}{*}{45.96} & & \\
\hline & A & 309151 & 7.49 & & & \\
\hline
\end{tabular}

* System 1: ethyl acetate/0.5\% phosphoric acid; 2: ethyl acetate/1.0\% phosphoric acid; 3: ethyl acetate/3.0\% phosphoric acid; 4 : ethyl acetate/5.0\% phosphoric acid; ${ }^{\mathrm{A}} \mathrm{C}$ : the organic phase; and ${ }^{\mathrm{B}} \mathrm{C}$ : the aqueous phase.

TABLE 2: The analysis results of the orthogonal experiment.

\begin{tabular}{|c|c|c|c|c|c|c|c|c|c|}
\hline No. & $V_{1}(\mathrm{ml})$ & $C_{1}(\mu \mathrm{g} / \mathrm{ml})$ & Extraction rate (\%) & $V_{2}(\mathrm{ml})$ & $V_{2}: V_{3}$ & $V_{3}(\mathrm{ml})$ & $C_{2}(\mu \mathrm{g} / \mathrm{ml})$ & LLE rate $(\%)$ & Transfer efficiency (\%) \\
\hline 1 & & & & & $1: 3$ & 12.0 & 5.36 & 99.03 & 86.89 \\
\hline 2 & 7 & 16.59 & 87.74 & 4.0 & $2: 3$ & 6.0 & 10.63 & 94.94 & 83.30 \\
\hline 3 & & & & & $4: 3$ & 3.0 & 18.88 & 91.27 & 80.08 \\
\hline 4 & & & & & $1: 3$ & 6.0 & 9.97 & 95.80 & 78.24 \\
\hline 5 & 3.5 & 30.91 & 81.67 & 2.0 & $2: 3$ & 3.0 & 20.23 & 104.64 & 85.46 \\
\hline 6 & & & & & $4: 3$ & 1.5 & 41.00 & 103.17 & 84.26 \\
\hline 7 & & & & & $1: 3$ & 3.0 & 17.84 & 179.33 & 77.65 \\
\hline 8 & 2.0 & 29.84 & 43.30 & 1.0 & $2: 3$ & 1.5 & 23.85 & 119.90 & 51.92 \\
\hline 9 & & & & & $4: 3$ & 0.75 & 48.85 & 122.78 & 53.16 \\
\hline
\end{tabular}

$C$ : the concentration of sibutramine hydrochloride in the detection solution. LLE rate: the extraction rate of liquid-liquid extraction step. Transfer efficiency: the efficiency of the sibutramine transfer from the sample to the test liquid.

TABLE 3: Effect of temperature on colorimetric reaction.

\begin{tabular}{lc}
\hline Temperature $\left({ }^{\circ} \mathrm{C}\right)$ & Phenomena \\
\hline 10 & No obvious pink insoluble substance forms within $30 \mathrm{~s}$ \\
15 & Pink insoluble substance forms within $30 \mathrm{~s}$ \\
20 & Pink insoluble substance forms within $30 \mathrm{~s}$ \\
25 & Pink insoluble substance forms within $30 \mathrm{~s}$ \\
\hline
\end{tabular}

13.63\% $(n=6)$; more details are demonstrated in Table 7. It was important to only gently shake the bottle to maintain easy operation, rapid results, and high reproducibility. We also used a shortened laying time. The recommended dosage of sibutramine hydrochloride (10 $\mathrm{mg}$ per dose), the content of the determined samples (1.90 $\mathrm{mg}$ to $67.02 \mathrm{mg}$ per capsule), and the transfer efficiency (above 30\%) give acceptable precision and high detection sensitivity $(0.025 \mathrm{mg} / \mathrm{ml}$, equal to $0.112 \mathrm{mg}$ per aliquot, see section LOD). This rapid screening method can determine whether sibutramine hydrochloride is added to NHM or DS products used for weight loss. 
TABLE 4: The results of determination of the samples.

\begin{tabular}{lcccc}
\hline Sample weight $(\mathrm{g})$ & Peak area A & $C^{1}(\mu \mathrm{g} / \mathrm{ml})$ & $X^{2}(\mathrm{mg} / \mathrm{g})$ & Average $(\mathrm{mg} / \mathrm{g})$ \\
\hline 0.10685 & 2828469 & 69.03 & 32.30 & \\
0.10835 & 2848987 & 69.53 & 32.09 & 32.21 \\
0.10627 & 2737640 & 66.81 & 31.43 & 2.58 \\
0.10711 & 2748151 & 67.07 & 31.31 & \\
0.10810 & 2884583 & 70.40 & 32.56 & \\
0.10192 & 2805760 & 68.47 & 33.59 & \\
\hline
\end{tabular}

${ }^{1} \mathrm{C}$ : the concentration of sibutramine hydrochloride in the detection solution, same as below. ${ }^{2} \mathrm{X}$ : the content of sibutramine hydrochloride in the test specimen.

TABLE 5: Results of the extraction.

\begin{tabular}{|c|c|c|c|c|c|}
\hline Sample weight (g) & Peak area A & $C(\mu \mathrm{g} / \mathrm{ml})$ & Extraction rate (\%) & Average (\%) & R.S.D. $\%(n=6)$ \\
\hline 0.10281 & 1045088 & 25.47 & 76.90 & \multirow{6}{*}{78.28} & \multirow{6}{*}{4.33} \\
\hline 0.10362 & 1031081 & 25.12 & 75.28 & & \\
\hline 0.10281 & 1067915 & 26.02 & 78.59 & & \\
\hline 0.10288 & 1042179 & 25.40 & 76.64 & & \\
\hline 0.10428 & 1169179 & 28.50 & 84.84 & & \\
\hline 0.10126 & 1036531 & 25.26 & 77.44 & & \\
\hline
\end{tabular}

TABLE 6: Results of liquid-liquid extaction rate.

\begin{tabular}{|c|c|c|c|c|c|}
\hline Content in solution $\mathrm{A}(\mathrm{mg})$ & Peak area $\mathrm{A}$ & $C(\mu \mathrm{g} / \mathrm{ml})$ & LLE rate $(\%)$ & Average (\%) & R.S.D. $\%(n=6)$ \\
\hline 0.9550 & 622675 & 15.15 & 47.59 & \multirow{6}{*}{59.45} & \multirow{6}{*}{11.01} \\
\hline 0.9422 & 799286 & 19.46 & 61.97 & & \\
\hline 0.9759 & 768581 & 18.71 & 57.52 & & \\
\hline 0.9523 & 779353 & 18.98 & 59.78 & & \\
\hline 1.0687 & 968675 & 23.60 & 66.25 & & \\
\hline 0.9472 & 824669 & 20.08 & 63.61 & & \\
\hline
\end{tabular}

TABLE 7: Calculation of transfer efficiency. The transfer efficiency is the product of the extraction rate and the LLE rate.

\begin{tabular}{lcccc}
\hline No. & Extraction rate (\%) & LLE rate (\%) & Transfer efficiency (\%) & Average (\%) \\
\hline 1 & 76.90 & 47.59 & 36.59 & \\
2 & 75.28 & 61.97 & 46.65 & 46.62 \\
3 & 78.59 & 57.52 & 45.21 & \\
4 & 76.64 & 59.78 & 45.81 & \\
5 & 84.84 & 66.25 & 56.21 & \\
6 & 77.44 & 63.61 & 49.26 & \\
\hline
\end{tabular}

3.4. Method Validation and Application. The purpose of this rapid screening method is to screen the samples for illegal addition of target analytes within about 3 minutes at the site of inspection. At the same time, dealing with large quantities of samples at low cost was also expected, this method generally consumed about a tenth the cost of conventional analysis methods. One out of it is to quickly screen out whether the sample is a positive one, and the positive rates of such samples are usually about $0 \sim 30 \%$ according to the data known. For negative results, no further instrumental analysis is required for confirmatory testing; for positive screening results, a conventional analytical method is used to test the sample to confirm the rapid screening results and accurately detect the content of the target component. The conventional analytical methods mentioned above are high-performance liquid chromatography with reference substances. The screening method has been validated according to Chinese Pharmacopoeia (Version 2010) 9101. These metrics include transfer efficiency, limit of detection (LOD), accuracy, sensitivity, and specificity via HPLC and comparison with the official methods approved by China Food and Drug Administration (CFDA) [42, 43].

3.4.1. LOD. We dissolved sibutramine hydrochloride in ethyl acetate and diluted it with ethyl acetate to obtain a series of concentrations of sibutramine hydrochloride (Table 8). Half of one Fufang Shoubao Capsule was transferred to $3.5 \mathrm{ml}$ of one of the sibutramine hydrochloride standard solutions to a bottle. The Fufang Shoubao product was also tested with an official method and found to be negative for sibutramine and thus was considered to be an interference. Testing was performed as described in the rapid screening 
TABLE 8: The determination of detection limit.

\begin{tabular}{lcc}
\hline Sample & Concentration of sibutramin hydrochloride $(\mathrm{mg} / \mathrm{ml})$ & Precipitate formed \\
\hline 1 & 0 & No \\
2 & 0.008 & No \\
3 & 0.010 & No \\
4 & 0.012 & Not sure \\
5 & 0.025 & Yes \\
6 & 0.050 & Yes \\
7 & 0.100 & Yes \\
\hline
\end{tabular}

When testing sibutramine within a dietary supplement, it was determined that $0.025 \mathrm{mg} / \mathrm{ml}$ sibutramine hydrochloride was the lowest visually detectable limit.

method procedure section, and the results are presented in Table 6. When the extraction rate (Table 5) is considered, the LOD in Table 6 will be adjusted based on equation (2). The result is $0.112 \mathrm{mg}$ of sibutramine per aliquot of sample (Table 9):

$$
\mathrm{LOD}_{\text {adjusted }}=\frac{\mathrm{LOD} \times 3.5}{78.28 \%} \text {. }
$$

3.4.2. Method Applications and Average Accuracy, Sensitivity, Specificity, and Selectivity. The rapid screening results can be true positive, false positive, true negative, or false negative versus the official methods approved by CFDA.

Accuracy, sensitivity, and specificity are the important parameters that characterized the precision of this method. These three parameters were calculated by the following equations, respectively:

$$
\begin{aligned}
& \text { accuracy }=\frac{\left(S-S_{F}\right)}{S} \times 100 \%, \\
& \text { sensitivity }=\frac{\left(P-N_{F}\right)}{P} \times 100 \%, \\
& \text { specificity }=\frac{\left(N-P_{F}\right)}{N} \times 100 \% .
\end{aligned}
$$

Here, $S$ is the total number of test specimens, $P_{T}$ is the number of the true positive specimens, $N_{T}$ is the number of the true negative specimens, $N_{F}$ is the number of the false negative specimens, $P_{F}$ is the number of the false positive specimens, $S_{F}$ is the summation of $P_{F}$ and $N_{F}, P$ is the summation of $P_{T}$ and $N_{F}$, and $N$ is the summation of $N_{T}$ and $P_{F}$.

Based on laboratory, civic, and provincial applications by different experimental level operators, the performance of the method is valuated below.

In our laboratory, we studied 60 batches of proprietary Chinese medicines and health foods that claimed an antiobesity function. These were purchased from three different cities. We compared the rapid screening results to the CFDA-approved method; the findings were completely consistent except for one false positive result (lab test results in Table 10).

With the assistance of two civic institutes of drug control, 47 batches of proprietary Chinese medicines and
Table 9: The determination of adjusted detection limit.

\begin{tabular}{lcc}
\hline Sample & $\begin{array}{c}\text { Content of sibutramin hydrochloride (mg } \\
\left.\text { per aliquot }{ }^{*}\right)\end{array}$ & $\begin{array}{c}\text { Precipitate } \\
\text { formed }\end{array}$ \\
\hline 1 & 0 & No \\
2 & 0.036 & No \\
3 & 0.045 & No \\
4 & 0.054 & Not sure \\
5 & 0.112 & Yes \\
6 & 0.224 & Yes \\
7 & 0.447 & Yes \\
\hline
\end{tabular}

According to the results listed in Table 9, the LOD is $0.112 \mathrm{mg}$ of sibutramine hydrochloride per aliquot for this method. The method is simple and rational. Aliquot: a certain amount of sample may vary from 0.1 to $0.5 \mathrm{~g}$.

health food sold for weight loss were purchased and examined by technicians at the two institutes. The results via the rapid screening method concurred with the CFDAapproved methods (small-scale results in Table 10).

In August 2008, the Guangdong province FDA launched a joint operation on antiobesity health food supervision in which 238 dealers were sampled, and 713 batches of weight loss DS were tested by the local FDA inspectors in the province who have a little experiment training. Two hundred and sixty-six brands of antiobesity health foods sold. Compared with the CFDA-approved method, only 7 out of the 713 samples were false positive by the rapid method (fullscale results in Table 10).

In this method, the target compound undergoes protonation, LLE, and a precipitation reaction (Figure 2). Each step offers selectivity and excludes interference substances. Therefore, the method produces high-accuracy results as shown above.

3.5. Verification and Evaluation from FDA. We requested the FDA division of pharmaceutical analysis to evaluate the rapid test kit in 2011. They prepared standards to estimate the detection limit of this test by spiking dietary supplements with sibutramine. They found that they could visually identify spiked dietary supplements with as little as $0.1 \mathrm{mg}$ of sibutramine. That is significant because a typical dose of sibutramine is $5 \mathrm{mg}$, so this detection limit is 50 times lower than the expected level of sibutramine in adulterated dietary supplements. They concluded that the rapid test kits are extremely useful to screen dietary supplements for the presence of sibutramine. 
TABLE 10: The primary technical parameters of this method.

\begin{tabular}{lccccccc}
\hline & $P_{T}$ & $N_{T}$ & $P_{F}$ & $N_{F}$ & Accuracy (\%) & Sensitivity (\%) & Specificity (\%) \\
\hline Lab test $(S=60)$ & 36 & 23 & 1 & 0 & 98.33 & 100 & 100 \\
Small-scale $(S=47)$ & 34 & 13 & 0 & 0 & 100 & 95.83 \\
Full-scale $(S=713)$ & 208 & 498 & 7 & 0 & 99.02 & 100 & 100 \\
Total $(S=820)$ & 278 & 534 & 8 & 0 & 99.02 & 100 & 98.61 \\
\hline
\end{tabular}

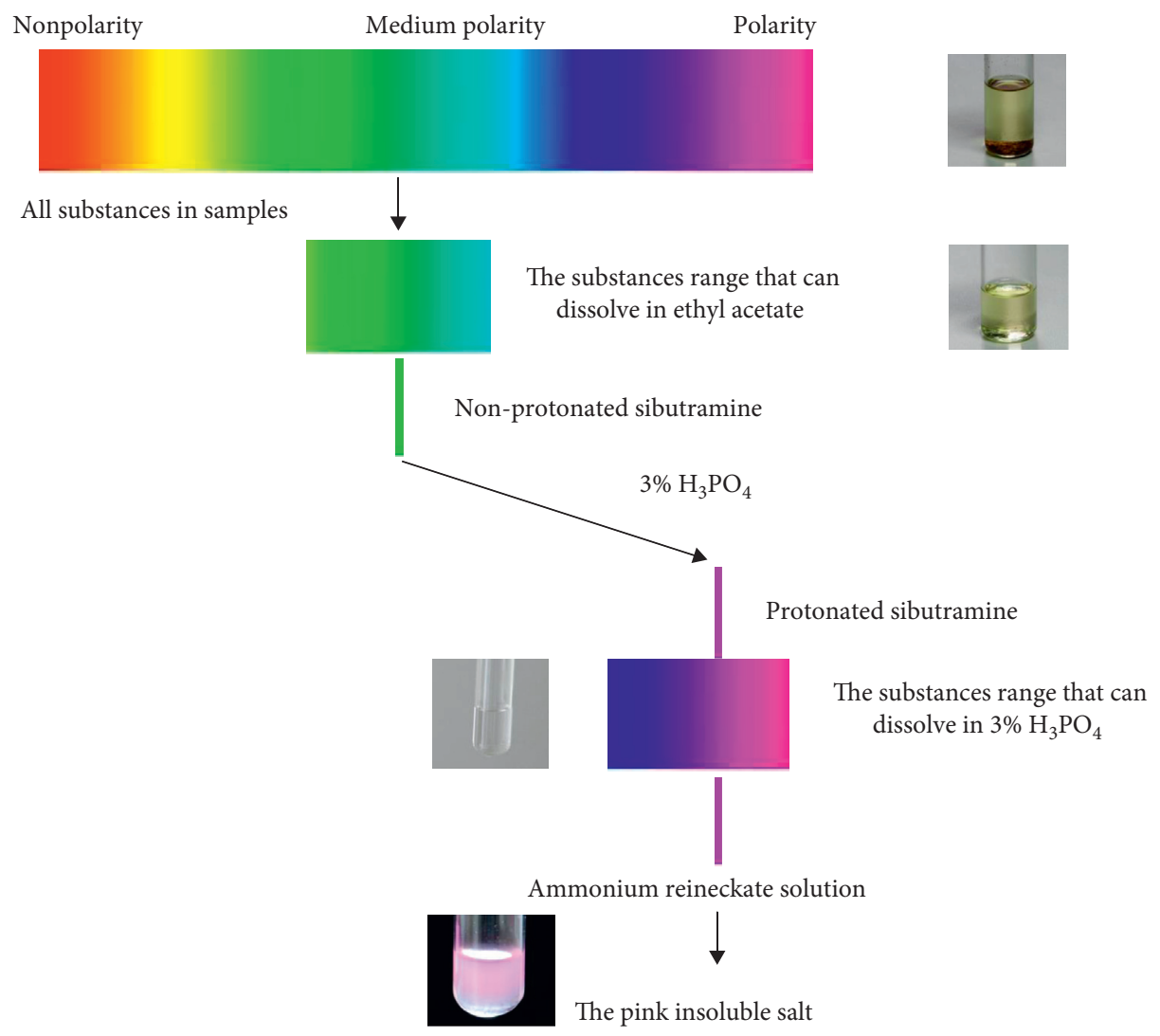

FIgURE 2: General schematic of the rapid screening method.

\section{Conclusions}

This study describes a new and easy on-site inspection method with high specificity, fast assay time, and low cost. This tool is likely to be used widely by local Food and Drug Administration personnel. The assay may overcome the shortcomings of current technology and solve the difficulties encountered in the supervision and administration of food and drugs to ensure public health safety.

This study uses a fast and novel colorimetric reaction. The methodology, choice of the reagent and test solution, evaluation model, LOD values, and verification steps described here are novel and may be useful to other researchers in the field.

While this rapid screening test is simple and easy to use, it does require a skilled operator and could not be used by common consumers without training. Thus, we are working on an improved version that can be employed by unskilled operators.

\section{Data Availability}

The data used to support the findings of this study are available from the corresponding author upon request.

\section{Disclosure}

The abstract was presented in "The 8th Global Summit on Regulatory Science Conference” (GSRS2018).

\section{Conflicts of Interest}

The authors declare no conflicts of interest regarding the publication of this paper.

\section{Acknowledgments}

This work was supported by the Bureau of Science and Information Technology of Guangzhou Municipal 
Government (2014J4100175), the Guangdong Medical Produces Administration (2018JDZ01), and Guangdong Quality and Efficacy Consistency Evaluation of Generic Drugs Research Platform (2017B020221002).

\section{References}

[1] C. Gundlah, K. F. Martin, D. J. Heal, and S. B. Auerbach, "In vivo criteria to differentiate monoamine reuptake inhibitors from releasing agents: sibutramine is a reuptake inhibitor," Journal of Pharmacology and Experimental Therapeutics, vol. 283, no. 2, pp. 581-591, 1997.

[2] F. Benazzi, "Organic hypomania secondary to sibutraminecitalopram interaction," Journal of Clinical Psychiatry, vol. 63, no. 2, p. 165, 2002.

[3] K. Binkle and S. R. Knowles, "Sibutramine and panic attacks," American Journal of Psychiatry, vol. 159, no. 10, pp. 1793-1794, 2002.

[4] Q. Cordeiro and H. Vallada, "Sibutramine-induced mania episode in a bipolar patient," The International Journal of Neuropsychopharmacology, vol. 5, no. 3, pp. 283-284, 2002.

[5] T. Sayin and M. Güldal, "Sibutramine: possible cause of a reversible cardiomyopathy," International Journal of Cardiology, vol. 99, no. 3, pp. 481-482, 2005.

[6] P. Fernández and A. M. Peiró, "A sibutramine-induced delusional disorder relapse," Journal of Neuropsychiatry and Clinical Neurosciences, vol. 19, no. 1, pp. 88-89, 2007.

[7] R. S. Padwal and S. R. Majumdar, "Drug treatments for obesity: orlistat, sibutramine, and rimonabant," Lancet, vol. 369, no. 9555, pp. 71-77, 2007.

[8] F. F. Da Rocha, T. O. Bamberg, F. C. C. Pinto, L. M. Guimarães e Gomes, and S. Silveira, "Hypomanic episode secondary to sibutramine in a patient with type-I bipolar disorder," Revista Brasileira De Psiquiatria, vol. 30, no. 4, pp. 400-401, 2009.

[9] B. Dogangun, N. Bolat, I. Rustamov, and L. Kayaalp, "Sibutramine-induced psychotic episode in an adolescent," Journal of Psychosomatic Research, vol. 65, no. 5, pp. 505-506, 2008.

[10] M. Florentin, E. N. Liberopoulos, and M. S. Elisaf, "Sibutramine-associated adverse effects: a practical guide for its safe use," Obesity Reviews, vol. 9, no. 4, pp. 378-387, 2008.

[11] J. Lee, T. Teoh, and T.-S. Lee, "Catatonia and psychosis associated with sibutramine: a case report and pathophysiologic correlation," Journal of Psychosomatic Research, vol. 64, no. 1, pp. 107-109, 2008.

[12] P. K. Fu, H. Y. Hsu, and P. Y. Wang, "Transient global amnesia after taking sibutramine: a case report," The Neurologist, vol. 16, no. 16, pp. 129-131, 2010.

[13] S. Naik, C. L. Khoo, R. Lua, S. B. Chai, A. Liew, and K. Sim, "Recurrent episodes of brief affective psychosis induced by sibutramine," Progress in Neuro Psychopharmacology \& Biological Psychiatry, vol. 34, no. 7, pp. 1359-1360, 2010.

[14] P. J. Nathan, B. V. O' Neill, and A. Napolitano, "Neuropsychiatric adverse effects of centrally acting antiobesity drugs," CNS Neuroscience and Therapeutics, vol. 17, no. 5, pp. 490-505, 2011.

[15] N. Waszkiewicz, B. Zalewska-Szajda, S. D. Szajda et al., "Sibutramine-induced mania as the first manifestation of bipolar disorder," BMC Psychiatry, vol. 12, no. 1, p. 43, 2012.

[16] [FDA] Food and Drug Administration, FDA Drug Safety Communication: FDA Recommends Against the Continued Use of Meridia (Sibutramine), U. S. Food and Drug Administration, Silver Spring, MD, USA, 2010.
[17] E. S. Simaremare, R. A. Susilowati, Y. D. Astuti et al., "Analysis of acetaminophen, mefenamic acid, sibutramine hydrochloride, and sildenafil citrate," Journal of Applied Pharmaceutical Science, vol. 8, no. 11, pp. 48-56, 2018.

[18] H. Hayun, B. P. Maggadani, and N. Amalina, "Determination of sibutramine adulterated in herbal slimming products using TLC densitometric method," Indonesian Journal of Pharmacy, vol. 27, no. 1, pp. 15-21, 2016.

[19] K. Marjan, H. Mehdi, K. Farzad et al., "Identification and determination of synthetic pharmaceuticals as adulterants in eight common herbal weight loss supplements," Iranian Red Crescent Medical Journal, vol. 16, no. 3, Article ID e1534, 2014.

[20] Y. P. Lin, Y. L. Lee, C. Y. Hung, C.-F. Chang, and Y. Chen, "Detection of adulterated drugs in traditional Chinese medicine and dietary supplements using hydrogen as a carrier gas," PLoS One, vol. 13, no. 10, Article ID e0205371, 2018.

[21] Y. Zhong, C. Sun, J. Xiong, and Y. Shi, "Simultaneous determination of eight adulterants in weight management supplements and herbs by HPLC-DAD and LC-MS/MS," Journal of Liquid Chromatography \& Related Technologies, vol. 40, no. 12, pp. 640-648, 2017.

[22] Z. Zaharieva, D. Tanev, and D. Danalev, "Development and validation of HPLC/DAD method for simultaneously determination of six prohibited substances in model matrices," Acta Chromatographica, vol. 32, no. 4, pp. 276-280, 2020.

[23] R. Jin, L. Li, L. Guo, W. Li, and Q. Shen, "A graphene tip coupled with liquid chromatography tandem mass spectrometry for the determination of four synthetic adulterants in slimming supplements," Food Chemistry, vol. 224, no. 1, pp. 329-334, 2017.

[24] Q. Cheng, L. Shou, C. Chen, S. Shi, and M. Zhou, "Application of ultra-high-performance liquid chromatography coupled with LTQ-orbitrap mass spectrometry for identification, confirmation and quantitation of illegal adulterated weightloss drugs in plant dietary supplements," Journal of Chromatography B, vol. 1064, pp. 92-99, 2017.

[25] P. Paula, M. J. E. Rodrigues, M. Correia, J. S. Amarlal, M. B. P. P. Oliveira, and C. Deleure-Matos, "Analysis of pharmaceutical adulterants in plant food supplements by UHPLC-MS/MS," European Journal of Pharmaceutical Sciences, vol. 99, no. 1, pp. 219-227, 2017.

[26] J. Hong, J. Hur, W. Lee et al., "Comprehensive screening of multiclass illegal adulterants in herbal supplements and spices using specific MS/MS fragmentations by UHPLC-Q/TOFMS," Analytical methods, vol. 11, no. 41, pp. 5260-5273, 2019.

[27] L. S. Müller, A. P. L. Moreira, D. T. Muratt, C. Viana, and L. M. de Carvalho, "An ultra-high performance liquid chromatography-electrospray tandem mass spectrometric method for screening and simultaneous determination of anorexic, anxiolytic, antidepressant, diuretic, laxative and stimulant drugs in dietary supplements marketed for weight loss," Journal of Chromatographic Science, vol. 57, no. 6, pp. 528-540, 2019.

[28] R. D. M. Thaís, D. S. Géssica, G. C. Leal et al., "A new approach to ion exchange cromatography with conductivity detection for adulterants investigation in dietary supplements," Biomedical Chromatography, vol. 33, no. 11, p. e4669, 2019.

[29] L. M. De Carvalho, P. A. Cohen, C. V. Silva et al., "A new approach to determining pharmacologic adulteration of herbal weight loss products," Food Additives \& Contaminants: Part A, vol. 29, no. 11, pp. 1661-1667, 2012.

[30] L. M. De Carvalho, M. Martini, A. P. Moreira, S. Garcia, P. Nascimento, and D. Bohrer, "Determination of synthetic pharmaceuticals in phytotherapeutics by capillary zone 
electrophoresis with contactless conductivity detection (CZEC4D)," Microchemical Journal, vol. 96, no. 1, pp. 114-119, 2010.

[31] R. Hachem, G. Assemat, N. Martins et al., "Proton NMR for detection, identification and quantification of adulterants in 160 herbal food supplements marketed for weight loss," Journal of Pharmaceutical \& Biomedical Analysis, vol. 124, pp. 34-47, 2016.

[32] J. D. Dunn, C. M. Gryniewicz-Ruzicka, D. J. Mans et al., "Qualitative screening for adulterants in weight-loss supplements by ion mobility spectrometry," Journal of Pharmaceutical \& Biomedical Analysis, vol. 71, pp. 18-26, 2012.

[33] N. Cebi, M. T. Yilmaz, and O. A. Sagdic, "A rapid ATR-FTIR spectroscopic method for detection of sibutramine adulteration in tea and coffee based on hierarchical cluster and principal component analyses," Food Chemistry, vol. 229, no. 15, pp. 517-526, 2017.

[34] Y. J. Xu and G. P. Xu, "Determination of sibutramine, indapamide, bumetanide and chlorothiazide in mass-reducing tonic by micellar electrokinetic capillary chromatography," Chinese Journal of Pharmaceutical Analysis, vol. 25, no. 12, pp. 1456-1459, 2005.

[35] K. Stypułkowsk, A. Błażewicz, J. Maurin, K. Sarna, and Z. Fijałek, "X-ray powder diffractometry and liquid chromatography studies of sibutramine and its analogues content in herbal dietary supplements," Journal of Pharmaceutical \& Biomedical Analysis, vol. 56, no. 5, pp. 969-975, 2011.

[36] K. Chitra, K. Sujatha, R. R. Sanjivkumar, A. Ghosh, and K. V. V. Kaushik, "Extractive colorimetric determination of sibutramine from its pharmaceutical preparation," Indian Drugs, vol. 41, pp. 478-481, 2004.

[37] R. A. Velapoldi and S. A. Wicks, "The use of chemical spot kits for the presumptive identification of narcotics and drugs of abuse," Journal of Forensic Sciences, vol. 19, no. 3, pp. 636-656, 1974.

[38] K. Miecznikowski, P. J. Kulesza, M. Ordak et al., "Color tests and electrocanalytical methods for the preliminary identification of drugs," The Electrochemical Society, 2016.

[39] National Institute of Standards and Technology (NIST) United States of America, Color Test Reagents/Kits for Preliminary Identification of Drugs of Abuse, Bureau of Justice Statistics, Washington, DC, USA, 2000.

[40] C. L. O’Neal, D. J. Crouch, and A. A. Fatah, "Validation of twelve chemical spot tests for the detection of drugs of abuse," Forensic Science International, vol. 109, no. 3, pp. 189-201, 2000.

[41] D. Lucero-Borja, O. Castilla, X. Subirats, and C. Ràfols, "Solubility-pH profiles of a free base and its salt: sibutramine as a case study," ADMET \& DMPK, vol. 5, no. 4, pp. 253-256, 2017.

[42] National Pharmacopoeia Committee of China, The Pharmacopoeia of the People's Republic of China, China Medical Science Press, Beijing, China, 2010.

[43] Z. Xie, Theories and Practices of Rapid Screening Technologies for Adulteration in Health Products, South China University of Technology Press, Guangzhou, China, 2011. 